\title{
STABILITY OF SINGULARITIES OF MINIMIZING HARMONIC MAPS
}

\author{
ROBERT HARDT \& FANG-HAU LIN
}

\section{Introduction}

Singularities of energy minimizing harmonic maps may occur with 3dimensional domains. Perhaps the simplest example is the map $x /|x|$ which has least energy [2] among all finite energy maps from the 3-ball $\mathbf{B}$ to the 2sphere $\mathbf{S}^{2}$ having boundary values given by the identity map of $\mathbf{S}^{2}$. Moreover, in dimension 3 , singularities are, by the work of R. Schoen and K. Uhlenbeck $[10$, Theorem], $[11,2.7]$, at most isolated. As the boundary data varies the singularities presumably move. In [5] was noted the impossibility of a sequence of minimizing configurations in which a pair of oppositely oriented singularities come together and cancel, leaving a singularity-free configuration. This followed from the strong convergence of minimizers and the basic small energy regularity theorem $[10,2.6]$. These arguments left open the possibility of three singularities, two oppositely oriented, merging and leaving a single singularity. This is not precluded by either topological degree considerations or by the monotonicity of energy $[11,2.4]$. However, the estimates of the present paper, in particular, rule out any such cancellation. Our results are based on the following:

Perturbation Lemma. There exist positive constants $\delta_{0}, c_{0}$, and $\alpha$ so that if $\varphi \in \operatorname{Lip}\left(\mathbf{S}^{2}, \mathbf{S}^{2}\right), \delta=\left\|\varphi-\mathrm{id}_{\mathbf{S}^{2}}\right\|_{\operatorname{Lip}} \leq \delta_{0}$, and $u \in H^{1}\left(\mathbf{B}, \mathbf{S}^{2}\right)$ is energy minimizing with $u \mid \mathbf{S}^{2} \equiv \varphi$, then $u$ has only one singular point $a$,

$$
|a| \leq c_{0} \delta^{1 / 2} \quad \text { and } \quad\left\|u-0\left(\frac{x-a}{|x-a|}\right)\right\|_{C^{\alpha}} \leq c_{0} \delta^{1 / 4}
$$

for some orthogonal rotation $\theta$ of $\mathbf{R}^{3}$ with $\left\|\theta-\mathrm{id}_{\mathbf{R}^{3}}\right\| \leq c_{0} \delta^{1 / 4}$.

This leads to the following general

Stability Theorem. Suppose $\Omega$ is a smooth bounded domain in $\mathbf{R}^{3}$, $\psi \in \operatorname{Lip}\left(\partial \Omega, \mathbf{S}^{2}\right)$, and $v$ is the unique energy-minimizing map from $\Omega$ to $\mathbf{S}^{2}$ with $v \mid \partial \Omega \equiv \psi$. There exists a positive number $\beta$ and, for any positive $\varepsilon, a$

Received June 26, 1987 and, in revised form, September 14, 1987. The authors' research was partially supported by the National Science Foundation. 
positive number $\delta$ so that for any $\varphi \in C^{1, \alpha}\left(\partial \Omega, \mathbf{S}^{2}\right)$ with $\|\varphi-\psi\|_{\text {Lip }} \leq \delta$ and for any energy-minimizing $u \in H^{1}\left(\Omega, \mathbf{S}^{2}\right)$ with $u \mid \partial \Omega \equiv \varphi$, one has

$$
\|u-v \circ \eta\|_{C^{\beta}} \leq \varepsilon
$$

for some bi-lipschitz transformation $\eta$ of $\Omega$ with $\left\|\eta-\mathrm{id}_{\Omega}\right\|_{\mathrm{Lip}} \leq \varepsilon$. In particular, $\eta$ maps the singularities of $u$ onto the singularities of $v$.

The uniqueness hypothesis may be obtained by restricting a minimizer to a proper subdomain. For examples of nonuniqueness, see [7], [1], and $\S 5$ below.

Our proof of these perturbation estimates is based on the regularity theory of energy minimizing maps ([10], [11], §2), the classification of minimizing tangent maps into $\mathbf{S}^{2}$ by H. Brezis, J. M. Coron, and E. Lieb [2] and asymptotic estimates of L. Simon [12], [13]. The latter works provide a description of the behavior of a minimizer in a punctured ball based only on information concerning its behavior on an annular region and its energy density at the center.

The Stability Lemma implies an interior estimate on the distance between singularities. Along with the uniform boundary estimate (2.1), it leads readily to global estimates on the number of singularities in terms of the Lipschitz norm of the boundary data. F. J. Almgren, Jr. and E. Lieb have in [1] independently obtained estimates in terms of the energy of the boundary data.

In our proof of the Perturbation Lemma, decay in an annulus is controlled by the boundary values. The proof easily generalizes to minimizers of other functionals as considered in [12]. In $\S 5$ we remark how the example of [8] leads to the existence of a smooth function $\varphi: \mathbf{S}^{2} \rightarrow \mathbf{S}^{2}$ which serves as boundary values for two distinct energy-minimizing maps, one having singularities and one completely free of singularities.

\section{Preliminary lemmas}

We will use $c_{0}, c_{1}, \ldots$ to denote universal constants, and let

$$
\mathbf{B}_{r}(a)=\left\{x \in \mathbf{R}^{3}:|x-a|<r\right\}, \quad \mathbf{B}_{r}=\mathbf{B}_{r}(0), \quad \mathbf{B}=\mathbf{B}_{1}(0), \quad \mathbf{S}^{2}=\partial \mathbf{B} .
$$

As in [9] we define, for any $C^{1}$ function $g: \mathbf{R}^{2} \rightarrow \mathbf{R}$ with $g(0)=0=|\nabla g(0)|$ and $\operatorname{Lip} g \leq 1$, the domain

$$
\Omega_{g}=\left\{\left(x_{1}, x_{2}, x_{3}\right) \in \mathbf{B}: x_{3}<g\left(x_{1}, x_{2}\right)\right\} .
$$

Lemma (Uniform boundary regularity). There exists positive numbers $\rho_{0}$ and $\sigma_{0}$ so that if $g$ is as above with $\operatorname{Lip} g \leq \sigma_{0}$ and if $u \in H^{1}\left(\Omega_{g}, \mathbf{S}^{2}\right)$ is energy 
minimizing with $\operatorname{Lip}\left(u \mid \mathbf{B} \cap \partial \Omega_{g}\right) \leq 1$, then $u \mid\left(\mathbf{B}_{\rho_{0}} \cap \bar{\Omega}_{g}\right)$ is Hölder continuous with

$$
\left\|u \mid\left(\mathbf{B}_{\rho_{0}} \cap \bar{\Omega}_{g}\right)\right\|_{C^{1 / 2}} \leq 1
$$

Proof. Recalling $[4,5.4,5.5]$, it suffices to find $\rho_{0}$ so that

$$
\rho_{0}^{-1} \int_{\mathbf{B}_{2 \rho_{0}} \cap \Omega_{g}}|\nabla u|^{2} d x \leq \alpha_{0}
$$

for some suitable positive constant $\alpha_{0}$. Before doing this we will establish an absolute bound

$$
\int_{\mathbf{B}_{1 / 2} \cap \Omega_{g}}|\nabla u|^{2} d x \leq c_{1} .
$$

This is analogous to the interior energy bound [6, 3.1].

First choose, as in [9, 5.2], uniformly bi-lipschitz maps $\Upsilon_{\sigma}: \mathbf{B}_{\sigma} \cap \Omega_{g} \rightarrow \mathbf{B}_{\sigma}$ for $\sigma \in\left[\frac{1}{2}, 1\right]$. Then select, as in [4, 2.3], an extension $\omega_{\sigma} \in H^{1}\left(\mathbf{B}_{\sigma}, \mathbf{S}^{2}\right)$ of $u \circ \Upsilon_{\sigma}^{-1}$ satisfying the estimate

$$
\int_{\mathbf{B}_{\sigma}}\left|\nabla \omega_{\sigma}\right|^{2} d x \leq c_{2}\left(\int_{\partial \mathbf{B}_{\sigma}}\left|\nabla_{\tan }\left(u \circ \Upsilon_{\sigma}^{-1}\right)\right|^{2} d s\right)^{1 / 2} .
$$

Transforming with $\Upsilon_{\sigma}$ and $\Upsilon_{\sigma}^{-1}$ and abbreviating

$$
\mathbf{D}(\sigma)=\int_{\mathbf{B}_{\sigma} \cap \Omega_{g}}|\nabla u|^{2} d x
$$

we conclude from the minimality of $u$ that, for almost all $\sigma \in\left[\frac{1}{2}, 1\right]$,

$$
\begin{aligned}
\mathbf{D}(\sigma) & \leq \int_{\mathbf{B}_{\sigma} \cap \Omega_{g}}\left|\nabla\left(\omega_{\sigma} \circ \Upsilon_{\sigma}\right)\right|^{2} d x \\
& \leq c_{3}\left(\int_{\mathbf{B}_{\sigma} \cap \partial \Omega_{g}}\left|\nabla_{\tan } u\right|^{2} d S+\int_{\partial \mathbf{B}_{\sigma} \cap \Omega_{g}}\left|\nabla_{\tan } u\right|^{2} d S\right)^{1 / 2} \\
& \leq c_{3}\left(\mathbf{D}^{\prime}(\sigma)+\sigma^{2}\right)^{1 / 2}
\end{aligned}
$$

for some $c_{3} \geq 1$. This implies that $\mathrm{D}\left(\frac{1}{2}\right) \leq 4 c_{3}^{2}$. In fact otherwise, for $\sigma \in\left[\frac{1}{2}, 1\right]$

$$
\begin{gathered}
\mathbf{D}(\sigma) \geq \mathbf{D}\left(\frac{1}{2}\right)>4 c^{2}>2 c_{3}, \\
\mathbf{D}^{\prime}(\sigma) \geq c_{3}^{-2} \mathbf{D}(\sigma)^{2}-\sigma^{-2} \geq c_{3}^{-2} \mathbf{D}(\sigma)^{2}-4 \geq \frac{1}{2} c_{3}^{-2} \mathbf{D}(\sigma)^{2},
\end{gathered}
$$

and we could integrate $-\mathbf{D}^{\prime} / \mathbf{D}^{2}$ from $\frac{1}{2}$ to 1 to find the contradiction

$$
-\mathbf{D}\left(\frac{1}{2}\right)^{-1} \leq \mathbf{D}(1)^{-1}-\mathbf{D}\left(\frac{1}{2}\right)^{-1} \leq-\frac{1}{4} c_{3}^{-2} \text { and } \mathbf{D}\left(\frac{1}{2}\right) \leq 4 c_{3}^{2} .
$$

Having established the bound (2), we now argue by compactness. If the lemma were false, then, by (1), there would exist sequences $\rho_{i} \rightarrow 0, \sigma_{i} \rightarrow 0$ 
and minimizers $u_{i} \in H^{1}\left(\Omega_{g_{i}}, \mathbf{S}^{2}\right)$ where $\operatorname{Lip} g_{i} \leq \sigma_{i}, \operatorname{Lip}\left(u_{i} \mid \mathbf{B} \cap \partial \Omega_{g_{i}}\right) \leq 1$, and

$$
\liminf _{i \rightarrow \infty} \rho_{i}^{-1} \int_{\mathbf{B}_{2 \rho_{i}} \cap \Omega_{g_{i}}}\left|\nabla u_{i}\right|^{2} d x \geq \alpha_{0} .
$$

By (2) (applied with $g(x)$ replaced by $g_{i}\left(2 \rho_{i} x\right)$ ) the sequence of scaled functions $v_{i}(x)=u_{i}\left(2 \rho_{i} x\right)$ have bounded energy on $\mathbf{B}$. A subsequence of the $v_{i}$ converge weakly in $H^{1}$ to a function $v \in H^{1}\left(\mathbf{B} \cap\left\{x_{3}>0\right\}, \mathbf{S}^{2}\right\}$ with $v \mid \mathrm{B} \cap\left\{x_{3}=0\right\} \equiv$ constant $\left(=\lim _{i \rightarrow \infty} u_{i}(0)\right)$. By $[9,6.4,5.7]$ and $[11,2.6], v$ must be a constant and the energies

$$
\left(2 \rho_{i}\right)^{-1} \int_{\mathbf{B}_{2 \rho_{i}} \cap \Omega_{g_{i}}}\left|\nabla u_{i}\right|^{2} d x=\int_{\mathbf{B} \cap\left(2 \rho_{i}\right)^{-1} \Omega_{g_{i}}}\left|\nabla u_{i}\right|^{2} d x
$$

converge to 0 , contradicting (3).

Lemma 2.2. There exist a positive constant $\delta_{0}$ so that if $\varphi \in \operatorname{Lip}\left(\mathbf{S}^{2}, \mathbf{S}^{2}\right)$, $\delta=\left\|\varphi-\mathrm{id}_{\mathbf{S}^{2}}\right\|_{\text {Lip }} \leq \delta_{0}$, and $u \in H^{1}\left(\mathbf{B}, \mathbf{S}^{2}\right)$ is energy minimizing with $u \mid \mathbf{S}^{2} \equiv \varphi$, then $u$ has only one singular point $a$ and $|a| \leq c_{4} \delta^{1 / 2}$.

Proof. First note that the minimality of such a $u$ implies the estimate

$$
\begin{aligned}
\int_{\mathbf{B}}|\nabla u|^{2} d x & \leq \int_{\mathbf{B}}\left|\nabla \varphi\left(\frac{x}{|x|}\right)\right|^{2} d x=\int_{\mathbf{S}^{2}}\left|\nabla_{\tan } \varphi\right|^{2} d s \\
& \leq \int_{\mathbf{B}}\left|\nabla\left(\frac{x}{|x|}\right)\right|^{2} d x+c_{5}\|\varphi-\mathrm{id}\|_{\text {Lip }}^{2} \\
& =8 \pi+c_{5}\|\varphi-\mathrm{id}\|_{\text {Lip }}^{2} .
\end{aligned}
$$

Assuming now that the lemma is false, we would find a sequence of energy minimizing maps $u_{i}: \mathbf{B} \rightarrow \mathbf{S}^{2}$ which do not satisfy the conclusion but which have Lipschitz boundary values $\varphi_{i}=u_{i} \mid \mathbf{S}^{2}$ so that

$$
\delta_{i}=\left\|\varphi_{i}-\operatorname{id}_{\mathbf{S}^{2}}\right\|_{\text {Lip }} \rightarrow 0 \quad \text { as } i \rightarrow \infty \text {. }
$$

By (4), any subsequence of the $u_{i}$ contains a subsequence that is weakly convergent in $H^{1}\left(\mathbf{B}, \mathbf{S}^{2}\right)$. Any limit function, having energy at most $8 \pi$ and having boundary values equalling $\mathrm{id}_{\mathbf{S}^{2}}$, must be $x /|x|[2]$. Thus, our original sequence $u_{i}$ converges to $x /|x|$ weakly, and, in fact, by $[10,4.6]$, strongly in $H^{1}$.

By Lemma 2.1, the functions are all uniformly Hölder continuous with uniformly bounded energies on some neighborhood of $\partial\left(\mathbf{B} \sim \mathbf{B}_{1 / 2}\right)$. For $i$ sufficiently large, $\varphi_{i}$ has degree one, and so $\operatorname{Sing}\left(u_{i}\right)$, the singular set of $u_{i}$, must be nonempty. Arguing as above using the interior regularity theory [10] and the Hölder continuity of $x /|x|$ away from the origin, we now find

$$
\sup \left\{|a|: a \in \operatorname{Sing}\left(u_{i}\right)\right\} \rightarrow 0 \quad \text { as } i \rightarrow \infty .
$$


Choose a point $a_{i} \in \operatorname{Sing}\left(u_{i}\right)$, let

$$
v_{i}: \mathbf{B}_{1-\left|a_{i}\right|} \rightarrow \mathbf{S}^{2}, \quad v_{i}(x)=u_{i}\left(x+a_{i}\right),
$$

and note, by the interior regularity theory, that

$$
\begin{aligned}
& \left\|v_{i}-\frac{x}{|x|}\right\|_{C^{2}\left(\mathbf{B}_{2 / 3}\left(a_{i}\right) \sim \mathbf{B}_{1 / 3}(a)\right)} \\
& \quad \leq\left\|u_{i}(x)-\frac{x}{|x|}\right\|_{C^{2}\left(\mathbf{B}_{3 / 4} \sim \mathbf{B}_{1 / 4}\right)}+\left\|\frac{x}{|x|}-\frac{x-a_{i}}{\left|x-a_{i}\right|}\right\|_{C^{2}\left(\mathbf{B}_{2 / 3} \sim \mathbf{B}_{1 / 3}\right)} \rightarrow 0
\end{aligned}
$$

as $i \rightarrow \infty$. As in the discussion in [12, §8], Theorem 1 of [12] is applicable to $v_{i}$. It implies that, for $i$ sufficiently large, 0 is the only singularity of $v_{i}$, hence $a_{i}$ is the only singularity of $u_{i}$.

Finally we need to estimate $\left|a_{i}\right|$. For this, we define the function $w_{i} \in$ $H^{1}\left(\mathbf{B}, \mathbf{S}^{2}\right)$,

$$
w_{i}(x)=u_{i}(2 x) \text { for } x \in \mathbf{B}_{1 / 2}, \quad w_{i}(x)=z_{i}(x) /\left|z_{i}(x)\right| \text { for } x \in \overline{\mathbf{B}} \sim \mathbf{B}_{1 / 2}
$$

where

$$
z_{i}(x)=(2-2|x|) \varphi_{i}\left(\frac{x}{|x|}\right)+(2|x|-1) \frac{x}{|x|}
$$

Noting that $\left\|\varphi_{i}-\mathrm{id}_{\mathbf{S}^{2}}\right\|_{L^{\infty}} \leq c_{6} \delta_{i}$, we readily compute that

$$
\begin{gathered}
\int_{\mathbf{B}}\left|\nabla w_{i}\right|^{2} d x-c_{7} \delta_{i} \leq \int_{\mathbf{B}_{1 / 2}}\left|\nabla u_{i}\right|^{2} d x+\int_{\mathbf{B}_{\mathbf{B}_{1 / 2}}}\left|\nabla\left(\frac{x}{|x|}\right)\right|^{2} d x \\
=\int_{\mathbf{B}_{1 / 2}}\left|\nabla u_{i}\right|^{2} d x+4 \pi=\int_{\mathbf{B}_{1 / 2}}\left|\nabla u_{i}\right|^{2} d x+2 \int_{1 / 2}^{1} \operatorname{Area~} u_{i}\left(\partial \mathbf{B}_{r}\right) d r \\
=\int_{\mathbf{B}_{1 / 2}}\left|\nabla u_{i}\right|^{2} d x+\int_{1 / 2}^{1} \int_{\partial \mathbf{B}_{r}}\left|\nabla_{\tan } u_{i}\right|^{2} d S d r \leq \int_{\mathbf{B}}\left|\nabla u_{i}\right|^{2} d x
\end{gathered}
$$

because degree $\left(u_{i} \mid \partial \mathbf{B}_{r}\right)=1$ for $\frac{1}{2} \leq r \leq 1$ and $i$ large enough to guarantee that degree $\left(\varphi_{i}\right)=1$ and $a_{i} \in \mathbf{B}_{1 / 2}$. Since $w_{i} \mid \mathbf{S}^{2} \equiv \mathrm{id}_{\mathbf{S}^{2}}$ and $w_{i}$ has a single degree one singularity at $a_{i}$, we may also infer, using [2], the energy lower bound

$$
\int_{\mathbf{B}}\left|\nabla w_{i}\right|^{2} d x \geq e \int_{\mathbf{S}^{2}}\left|\omega-a_{i}\right| d S \omega \geq 8 \pi+c_{8}\left|a_{i}\right|^{2} .
$$


Combining this inequality with (4) and (5), we obtain the estimate

$$
\left|a_{i}\right| \leq c_{4} \delta_{i}^{1 / 2}
$$

which contradicts the original choice of $u_{i}$.

\section{Proof of the Perturbation Lemma}

By Lemma 2.2, $u$ has, for $\delta$ sufficiently small, a single singularity $a$ with $|a| \leq c_{4} \delta^{1 / 2}$. From [2] we infer that

$$
\underset{r \downarrow 0}{\limsup } r^{-1} \int_{\mathbf{B}_{r}(a)}|\nabla u|^{2} d x=8 \pi
$$

and that any tangent map [10] of $u$ at $a$ must be in the form $\theta(x /|x|)$ for some rotation $\theta$ of $\mathbf{R}^{3}$. Theorem 1 of [12] implies that $\theta$ is unique and that

$$
A(r)=\left(\|(\partial / \partial r) v(r \cdot)\|_{C^{1}\left(\mathbf{S}^{2}\right)}+\|v(r \cdot)-\theta\|_{C^{2}\left(\mathbf{S}^{2}\right)}\right) \rightarrow 0 \text { as } r \rightarrow 0
$$

where $v(x)=u(x+a)$. Moreover, by the integrability of the Jacobi fields of $x /|x|[3],[12$, Theorem 1] may here be replaced by the more elementary alternative argument of $[13, \S 6]$. The latter implies, for some positive $\beta<1$ and $\delta$ sufficiently small, the estimates

$$
\left\|\theta-\operatorname{id}_{\mathbf{R}^{3}}\right\| \leq c_{q} \varepsilon \text { and } A(r) \leq c_{q} \varepsilon r^{\beta}
$$

where $\varepsilon=\|v(x)-x /|x|\|_{C^{2}\left(\mathbf{B}_{2 / 3} \sim \mathbf{B}_{1 / 3}\right)}$. In particular, for any positive $\alpha<\beta$,

$$
\left\|u-\theta\left(\frac{x-a}{|x-a|}\right)\right\|_{C^{\alpha}\left(\mathbf{B}_{1 / 2}\right)} \leq c_{10} \varepsilon .
$$

Since, by (6) and the definitions of $\delta$ and $\varepsilon$,

$$
\begin{aligned}
& \left\|u-\theta\left(\frac{x-a}{|x-a|}\right)\right\|_{\operatorname{Lip} \partial\left(\mathbf{B} \sim \mathbf{B}_{1 / 2}\right)} \\
& \quad \leq c_{11}(\varepsilon+|a|)+\delta+\left\|u-\theta\left(\frac{x-a}{|x-a|}\right)\right\|_{\operatorname{Lip} \partial\left(\mathbf{B}_{1 / 2}\right)} \leq c_{12}\left(\varepsilon+\delta^{1 / 2}\right),
\end{aligned}
$$

standard interior and boundary estimates also imply that 


$$
\left\|u-\theta\left(\frac{x-a}{|x-a|}\right)\right\|_{C^{1 / 2}\left(\mathbf{B} \sim \mathbf{B}_{1 / 2}\right)} \leq c_{13}\left(\varepsilon+\delta^{1 / 2}\right)
$$

for $\delta$ sufficiently small. By (7) and (8) it only remains to estimate $\varepsilon$.

For $1 / 8 \leq|x| \leq 1$, we find by integration radially and Schwarz's inequality that

$$
\begin{aligned}
\left|u(x)-\frac{x}{|x|}\right| & \leq\left|\varphi\left(\frac{x}{|x|}\right)-\frac{x}{|x|}\right|+\left|\int_{|x|}^{1}\left(\frac{\partial u}{\partial \rho}\right)\left(\rho \frac{x}{|x|}\right) d \rho\right| \\
& \leq\|\varphi-\mathrm{id}\|_{L^{\infty}}+\left(\int_{1 / 8}^{1}\left(\frac{\partial u}{\partial \rho}\right)^{2}\left(\rho \frac{x}{|x|}\right) d \rho\right)^{1 / 2} . \\
& \leq\|\varphi-\mathrm{id}\|_{L^{\infty}}+\left(64 \int_{1 / 8}^{1}\left(\frac{\partial u}{\partial \rho}\right)^{2}\left(\rho \frac{x}{|x|}\right) \rho^{2} d \rho\right)^{1 / 2} .
\end{aligned}
$$

Squaring and integrating give

(9)

$$
\begin{aligned}
\int_{\mathbf{B} \sim \mathbf{B}_{1 / 8}}\left|u(x)-\frac{x}{|x|}\right|^{2} d x & \leq 8 \pi\|\varphi-\mathrm{id}\|_{L^{\infty}}^{2}+512 \pi \int_{\mathbf{B} \sim \mathbf{B}_{1 / 8}}\left(\frac{\partial u}{\partial \rho}\right)^{2} d x \\
& \leq 8 \pi^{3}\|\varphi-\mathrm{id}\|_{L_{\text {ip }}}^{2}+512 \pi \int_{\mathbf{B} \sim \mathbf{B}_{1 / 8}}\left(\frac{\partial u}{\partial \rho}\right)^{2} d x
\end{aligned}
$$

because $\varphi(\omega)=\omega$ for some $\omega \in \mathbf{S}^{2}$. To estimate the latter term we will use the monotonicity equality $[10,2.4],[9,4.1]$ on the annulus $\mathbf{B} \sim \mathbf{B}_{1 / 8}$ and on the ball $\mathbf{B}_{1 / 8-|a|}(a) \subset \mathbf{B}_{1 / 8}$ to find that

$$
\begin{aligned}
\int_{\mathbf{B} \sim \mathbf{B}_{1 / 8}} & \left(\frac{\partial u}{\partial \rho}\right)^{2} d x \leq \int_{1 / 8}^{1}\left(2 \rho^{-1} \int_{\partial \mathbf{B}_{\rho}}\left(\frac{\partial u}{\partial \rho}\right)^{2} d S\right) d \rho \\
& =\int_{\mathbf{B}}|\nabla u|^{2} d x-8 \int_{\mathbf{B}_{1 / 8}}|\nabla u|^{2} d x \\
& \leq \int_{\mathbf{B}}|\nabla u|^{2} d x-(1-8|a|)\left[\frac{8}{1-8|a|}\right] \int_{\mathbf{B}_{1 / 8-|a|}(a)}|\nabla u|^{2} d x \\
& \leq \int_{\mathbf{B}}|\nabla u|^{2} d x-(1-8|a|) \lim _{\rho \rightarrow 0} \rho^{-1} \int_{\mathbf{B}_{\rho}(a)}|\nabla u|^{2} d x \\
& \leq \int_{\mathbf{B}}|\nabla u|^{2} d x-(1-8|a|) 8 \pi .
\end{aligned}
$$

From this inequality, (9), (4), and 2.2 we now deduce that

$$
\int_{\mathbf{B} \sim \mathbf{B}_{1 / 8}}\left|u(x)-\frac{x}{|x|}\right|^{2} d x \leq 8 \pi^{3} \delta^{2}+c_{5} \delta^{2}+64 \pi c_{4} \delta^{1 / 2} .
$$


Hence, by standard interior estimates $[12, \S 1]$,

$$
\left\|u(x)-\frac{x}{|x|}\right\|_{C^{2}\left(\mathbf{B}_{3 / 4} \sim \mathbf{B}_{1 / 4}\right)} \leq c_{14} \delta^{1 / 4}
$$

for $\delta$ sufficiently small. Thus,

$$
\begin{aligned}
\varepsilon & =\left\|v(x)-\frac{x}{|x|}\right\|_{C^{2}\left(\mathbf{B}_{2 / 3} \sim \mathbf{B}_{1 / 3}\right)}+\left\|\frac{x}{|x|}-\frac{x-a}{|x-a|}\right\|_{C^{2}\left(\mathbf{B}_{2 / 3} \sim \mathbf{B}_{1 / 3}\right)} \\
& \leq\left\|u(x)-\frac{x}{|x|}\right\|_{C^{2}\left(\mathbf{B}_{3 / 4} \sim \mathbf{B}_{1 / 4}\right)}+ \\
& \leq c_{15} \delta^{1 / 4}+c_{16}|a| \leq c_{17} \delta^{1 / 4},
\end{aligned}
$$

which, along with (7) and (8), completes the proof.

\section{Proof of the Stability Theorem}

Assume that $u_{i}$ is a sequence of energy minimizing maps from $\Omega$ to $\mathbf{S}^{2}$ which have Lipschitz boundary values $\varphi_{i}=u_{i} \mid \partial \Omega$ with

$$
\left\|\varphi_{i}-\psi\right\|_{\text {Lip }} \rightarrow \text { as } i \rightarrow \infty \text {. }
$$

First we note that the energies $\int_{\Omega}\left|\nabla u_{i}\right|^{2} d x$ are bounded. In fact the harmonic vectors $h_{i}: \bar{\Omega} \rightarrow \mathbf{R}^{3}$ with $h_{i} \mid \partial \Omega=\varphi_{i}$ clearly have bounded energies. The same holds for the maps $w_{i}$ obtained by suitably projecting, as in $[9,6.2]$, the $h_{i}$ onto $\mathbf{S}^{2}$. Moreover,

$$
\int_{\Omega}\left|\nabla u_{i}\right|^{2} d x \leq \int_{\Omega}\left|\nabla w_{i}\right|^{2} d x
$$

by minimality.

Next we reason as in the proof of the Stability Lemma and use the uniqueness of $v$ to deduce that the functions $u_{i}$ converge strongly in $H^{1}$ to $v$. By $[10$, Theorem II], $[11,2.7]$ the set of singularities of $v$ is a finite (possibly empty) subset $A=\left\{a_{1}, a_{2}, \cdots, a_{k}\right\}$ of $\Omega$.

For each singularity $a_{j}$, we infer from [10], [13], and [2] that there is a unique tangent map of $v$ at $a_{j}$ in the form $\theta_{j}\left(\frac{x}{|x|}\right)$ for some rotation $\theta_{j}$ of $\mathbf{R}^{3}$. As in the proof of the Perturbation Lemma, we may by [12, Theorem 1] or $[3],[13, \S 6]$ find a fixed positive constants $\tau$ and $\gamma$ (depending on $v$ ) so that

$$
\tau<\frac{1}{2} \min _{j} \operatorname{dist}\left(a_{j},\left(A \sim\left\{a_{j}\right\}\right) \cup \partial \Omega\right\}
$$




$$
\begin{gathered}
\left\|v-\theta_{j}\left(\frac{x-a_{j}}{\left|x-a_{j}\right|}\right)\right\|_{C^{2}\left(\mathbf{B}_{3 \tau / 2}\left(a_{j}\right) \sim \mathbf{B}_{\tau / 2}\left(a_{j}\right)\right)} \leq \frac{1}{2} \delta_{0}, \\
\left\|v-\theta\left(\frac{x-a_{j}}{\left|x-a_{j}\right|}\right)\right\|_{C^{\alpha}\left(\mathbf{B}_{\rho}\left(a_{j}\right)\right)} \leq \gamma \rho^{1 / 2 \alpha} .
\end{gathered}
$$

The uniqueness of $v$ and a compactness argument now show that

$$
\delta_{i}=\left\|u_{i}-v\right\|_{\operatorname{Lip}\left(\partial \mathbf{B}_{\tau}\left(a_{j}\right)\right)} \rightarrow 0 \quad \text { as } i \rightarrow \infty .
$$

As in the proof of the Stability Lemma, the uniform boundary regularity Lemma 2.1, and the interior regularity theory now gives the estimate

$$
\left\|u_{i}-v\right\|_{C^{\alpha}\left(\Omega \sim \bigcup_{j=1}^{k} \mathbf{B}_{\tau}\left(a_{j}\right)\right)} \leq c_{18} \delta_{i} \text { for } \delta_{i} \leq c_{18}^{-1} .
$$

By (10) and (12) we may, for $i$ sufficiently large, translate,rotate, and scale to apply the Perturbation Lemma. We obtain single points $a_{j i} \in \mathbf{B}_{\tau}\left(a_{j}\right)$ and rotations $\theta_{j i}$ of $\mathbf{R}^{3}$ so that

$$
\left|a_{j i}-a_{j}\right|+\left\|\theta_{j i}-\theta_{j}\right\|+\left\|u_{i}-\theta_{j i}\left(\frac{x-a_{j i}}{\left|x-a_{j i}\right|}\right)\right\|_{C^{\alpha}\left(\mathbf{B}_{\tau}\left(a_{j}\right)\right)} \leq c_{19} \delta_{i}^{1 / 4} .
$$

We now let

$$
\tau_{i}=\max _{j}\left|a_{j i}-a_{j}\right|^{1 / 2} \leq c_{20}^{1 / 2} \delta_{i}^{1 / 8},
$$

and define $\eta_{i}: \Omega \rightarrow \Omega$ so that

$$
\begin{aligned}
& \eta_{i}(x)=x \quad \text { on } \Omega \sim \bigcup_{j=1}^{k} \mathbf{B}_{\tau_{i}}\left(a_{j}\right), \\
& \eta_{i}(x)=\lambda_{j i}(x) \xi_{j i}(x)+\left[1-\lambda_{j i}(x)\right] x \quad \text { on } \mathbf{B}_{\tau_{i}}\left(a_{j}\right),
\end{aligned}
$$

where

$$
\begin{aligned}
& \xi_{j i}(x)=\theta_{j}^{-1} \theta_{j i}\left(x-a_{j i}\right)+a_{j}, \\
& \lambda_{j i} \in C^{\infty}(\Omega,[0,1]), \quad\left|\nabla \lambda_{j i}\right| \leq \tau_{i} / 3, \\
& \lambda_{j i} \equiv 1 \quad \text { on } \mathbf{B}_{\tau_{i} / 2}\left(a_{j}\right), \quad \text { and } \quad \lambda_{j i} \equiv 0 \quad \text { on } \Omega \sim \mathbf{B}_{\tau_{i}}\left(a_{j}\right) .
\end{aligned}
$$

Using (11), (13), (14), and (15), we now conclude that

$$
\left\|\eta_{i}-\operatorname{id}_{\Omega}\right\|_{\text {Lip }} \leq c_{20} \delta_{i}^{1 / 8} \text { and }\left\|u_{i}-v \circ \eta_{i}\right\|_{C^{\beta}} \leq c_{20} \delta_{i}^{1 / 4}
$$

for any positive $\beta<\frac{1}{2} \alpha$ and $i$ sufficiently large. 


\section{Remark}

An easy consequence of the Stability Theorem is that

the number of singularities of an energy-minimizing map $u \in H^{1}\left(\Omega, \mathbf{S}^{2}\right)$ is bounded by a constant $C$, depending only on $\Omega$ and $\|u \mid \partial \Omega\|_{\text {Lip }}$.

To see this one infers by scaling and 2.1 that there is, for each positive $L$, a positive number $\delta=\delta(\Omega, L)$ so that any energy-minimizing map $u \in$ $H^{1}\left(\Omega, \mathbf{S}^{2}\right)$ with $\|u \mid \partial \Omega\|_{\text {Lip }} \leq L$ has no singularities on $\Omega \sim \Omega_{\delta}$ where $\Omega_{\delta}=$ $\{x \in \Omega: \operatorname{dist}(x, \partial \Omega)>\delta\}$. On the other hand, any sequence of energyminimizing maps $u_{i} \in H^{1}\left(\Omega, \mathbf{S}^{2}\right)$ has, by the universal interior energy bound of $[6,3.1]$, a subsequence which converges in $H_{\text {loc }}^{1}$ to a map $u_{0} \in H_{\text {loc }}^{1}\left(\Omega, \mathbf{S}^{2}\right)$ which minimizes energy on each compact subset of $\Omega$. In particular $u_{0}$ has only a finite number of singularities in $\Omega_{\delta}$. By the Stability Theorem, $u_{i}$ has, for $i$ sufficiently large, precisely the same number of singularities in $\Omega_{\delta}$.

\section{An example of nonuniqueness}

There exists a smooth function $\varphi: \mathbf{S}^{2} \rightarrow \mathbf{S}^{2}$ which serves as boundary data for two energy minimizing maps from $\mathbf{B}^{3}$ to $\mathbf{S}^{2}$, one having no singularities and one having at least two singularities.

Here one may choose as in [8] a smooth function $\psi: \mathbf{S}^{2} \rightarrow \mathbf{S}^{2}$ of degree 0 so that any energy-minimizing map with boundary data $\psi$ must have at least two singularities. There is a smooth family of smooth functions $\varphi_{t}: \mathbf{S}^{2} \rightarrow \mathbf{S}^{2}$ for $0 \leq t \leq 1$, so that $\varphi_{1} \equiv \psi$ and $\varphi_{0}$ is a constant function. For all sufficiently small positive $t$, any energy-minimizer with boundary data $\varphi_{t}$ must, by the regularity theory [10], [11], be free of singularities. Let

$\tau=\sup \{t:$ every energy-minimizer with boundary data

$$
\left.\varphi_{t} \text { has no singularities }\right\} \text {. }
$$

Then $0<\tau<1$. Choose a sequence $s_{i} \uparrow \tau$ and singularity-free, energyminimizing maps $f_{i} \in H^{1}\left(\mathbf{B}, \mathbf{S}^{2}\right)$ with $f_{i} \mid \mathbf{S}^{2}=\varphi_{t_{i}}$. Also choose a sequence $t_{i} \downarrow \tau$ (possibly all $t_{i}=\tau$ ) and singular energy-minimizing maps $g_{i} \in H^{1}\left(\mathbf{B}, \mathbf{S}^{2}\right)$ with $g_{i} \mid \mathbf{S}^{2}=\varphi_{t_{i}}$. Passing to subsequences, without changing notation, $f_{i}$ and $g_{i}$ converge in $H^{1}\left(\mathbf{B}, \mathbf{S}^{2}\right)$ to energy-minimizing maps $f$ and $g$ with $f\left|\mathbf{S}^{2}=\varphi_{\tau}=g\right| \mathbf{S}^{2}$.

The map $f$ has no singularities. In fact, any possible singularity $a$ must, by [10], [11], occur on the interior. For a small positive $\rho, f \mid \partial \mathbf{B}_{\rho}(a)$ is smooth and of nonzero degree. The same would hold for $f_{i}$, for $i$ sufficiently large, by the interior regularity theory. But this would contradict the continuity of $f_{i} \mid \overline{\mathbf{B}_{\rho}(a)}$. 
Finally the map $g$ must have (interior) singularities because otherwise the regularity theory would imply the smoothness of $g_{i}$, for $i$ sufficiently large.

\section{References}

[1] F. J. Almgren, Jr. \& E. Lieb, Minimizing harmonic mappings: bounds on the number of singularities and examples (including symmetry breaking), preprint.

[2] H. Brezis, J. M. Coron \& E. Lieb, Harmonic maps with defects, Comm. Math. Phys. 107 (1986) 649705.

[3] R. Gulliver \& B. White, The rate of convergence of a harmonic map at a singular point, to appear in Math. Ann.

[4] R. Hardt, D. Kinderlehrer \& F. H. Lin, Existence and partial regularity of static liquid crystal configurations, Comm. Math. Phys. 105 (1986) 547-570.

[5] __, A remark about the stability of smooth equilibrium configurations of static liquid crystals, Mol. Crys. Liq. Cryst. 139 (1986) 189-194.

[6] _ Stable defects of minimizers of constrained variational problems, to appear in Anal. Nonlineare, Ann. Inst. Henri Poincaré.

[7] __ Instable configurations of static liquid crystals, in preparation.

[8] R. Hardt \& F. H. Lin, A remark on $H^{1}$ mappings, Manuscripta Math. 56 (1986) 1-10.

[9] _ Mappings minimizing the $L^{p}$ norm of the gradient, Comm. Pure Appl. Math. 40 (1987) 555-588.

[10] R. M. Schoen \& K. Uhlenbeck, A regularity theory for harmonic maps, J. Differential Geometry 17 (1982) 307-335.

[11] _ Boundary regularity and the Dirichlet problem of harmonic maps, J. Differential Geometry 18 (1983) 253-268.

[12] L. Simon, Asymptotics for a class of nonlinear evolution equations, with applications to geometric problems, Ann. of Math. (2) 118 (1983) 525-571.

[13] _ Isolated singularities of extrema of geometric variational problems, Lecture Notes in Math., Vol. 1161, Springer, Berlin, 1986.

UNIVERSITY OF MINNESOTA

NEW YORK UNIVERSITY 
